\title{
Magnetic monopole searches with the MoEDAL experiment at the LHC
}

\author{
J. Pinfold and R. Soluk \\ Physics Department, University of Alberta, Edmonton, Alberta, Canada T6G 0V1 \\ D. Lacarrère
}

Physics Department, CERN, 1211 Geneva 23, Switzerland

\section{P. Mermod* and A. Katre}

Particle Physics department, University of Geneva, 1211 Geneva 4, Switzerland

E-mail: philippe.mermodecern.ch

\begin{abstract}
The magnetic monopole appears in theories of spontaneous gauge symmetry breaking and its existence would explain the quantisation of electric charge. MoEDAL is the latest approved LHC experiment, designed to search directly for monopoles. It has now taken data for the first time. The MoEDAL detectors are based on two complementary techniques: nuclear-track detectors are sensitive to the high-ionisation signature expected from a monopole, and the new magnetic monopole trapper (MMT) relies on the stopping and trapping of monopoles inside an aluminium array which is then analysed with a superconducting magnetometer. Preliminary results obtained with a subset of the MoEDAL MMT test array deployed in 2012 are presented, where monopoles with charge above the fundamental unit magnetic charge or mass above $1.5 \mathrm{TeV}$ are probed for the first time at the LHC.
\end{abstract}

The European Physical Society Conference on High Energy Physics -EPS-HEP2013

18-24 July 2013

Stockholm, Sweden

\footnotetext{
*Speaker.
} 


\section{Introduction}

The existence of monopoles would add symmetry to Maxwell's equations of electromagnetism. In 1931 Dirac showed that electric charge quantisation could be explained as a natural consequence of angular momentum quantisation if one assumes the existence of magnetic monopoles [1]. In 1974, 't Hooft and Polyakov independently demonstrated that a GUT scheme with the $\mathrm{U}(1)$ subgroup of electromagnetism embedded into a larger gauge group which becomes spontaneously broken by the Higgs mechanism automatically possessed a topological monopole solution [2,3]. It has also been proposed [4] that monopole solutions could arise within the electroweak theory itself, which also relies on spontaneous gauge symmetry breaking. This so-called electroweak monopole would have a mass of the order of several $\mathrm{TeV}$ [5], possibly within reach of the LHC. From an experimental point of view, these considerations are compelling motivations for searching for free magnetic charges in nature, and the monopole mass is treated as a free parameter.

It follows from Dirac's argument that monopoles should carry a magnetic charge $g$ equal to a multiple of a fundamental unit of magnetic charge referred to as the Dirac charge $g_{D}: g=n$. $g_{D}$, where $g_{D}$ is equivalent to 68.5 times the charge of an electron. The minimum value of the quantisation number is $n=1$ according to Dirac or $n=2$ according to Schwinger [6], and $n=3$ or $n=6$ if one considers the elementary charge to be carried by the down-quark. As a result of the high value of the Dirac magnetic charge, a high-velocity monopole is expected to suffer energy losses in matter over 4500 times higher than a muon [7, 8, 9]. Monopoles should thus generally manifest themselves as very highly ionising particles. Another way to identify a free magnetic charge is to measure the persistent current it would induce when passed through a superconducting loop. Searches for monopoles using these two signatures (high ionisation and magnetic induction) were performed in cosmic rays and in matter, and at accelerators each time a new energy regime was reached [10]. The fact that no solid evidence for their existence has been gathered so far in so many experiments remains a mystery.

At colliders, three kinds of techniques are commonly used: (1) General-purpose detectors with high ionisation energy loss detection capabilities (e.g. OPAL at LEP [11] and CDF at the Tevatron [12]); (2) dedicated deployment of nuclear-track detectors [13] around the interaction points (e.g. at LEP [14, 15] and at the Tevatron [16]); and (3) the induction technique applied to accelerator and detector material in which monopoles may have stopped and remained trapped (e.g. at HERA [17] and at the Tevatron [18, 19]). Together, these searches excluded the presence of monopoles with charge equal to or above the Dirac charge and masses up to $400 \mathrm{GeV}$. Masses higher by one order of magnitude (up to $4 \mathrm{TeV}$ ) can be probed at the LHC. For optimum results, the LHC programme should include all three of these techniques [20]. An initial monopole search was performed at the ATLAS general-purpose experiment [21]. Monopole trapping experiments were shown to be feasible at the LHC [22]. The dedicated MoEDAL experiment near the LHCb interaction point uses a combination of in-flight detection with nuclear-track detectors [23] and trapping with aluminium absorbers. In this paper, we present preliminary data from a small part (9\%) of the MoEDAL magnetic monopole trapper (MMT) test array deployed in 2012. 

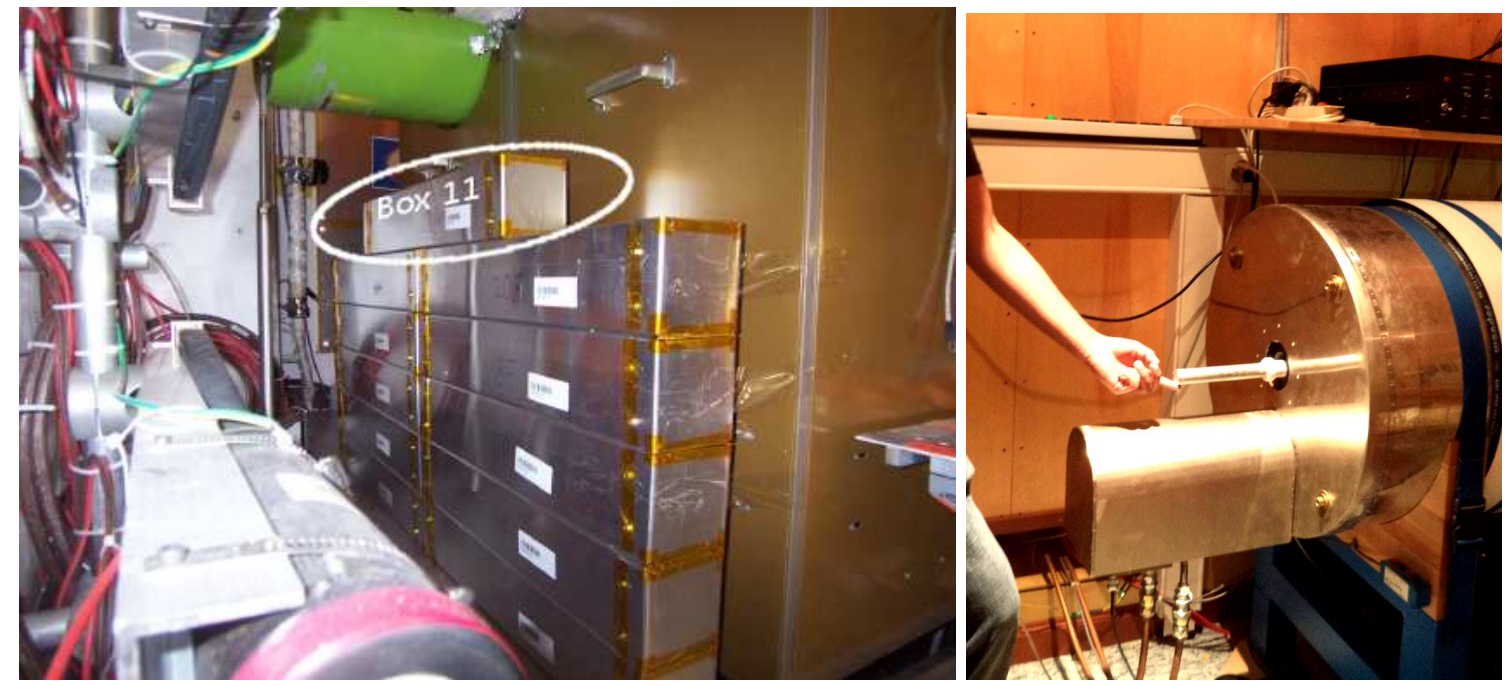

Figure 1: Left: Photograph of the 2012 MMT test array stacked under the beam pipe $1.8 \mathrm{~m}$ away from the interaction point along the back of the VELO vacuum vessel on the side opposite to the LHCb detector. The box which was analysed for this study (Box 11) is indicated. Right: Photograph of a sample attached to its holder at the starting position, just before passing through the magnetometer.

\section{Experimental procedure}

The MoEDAL MMT is an aluminium volume placed in the vicinity of the LHCb interaction point. The choice of material is driven by several factors: aluminium is cheap, non-magnetic, and has a nucleus which does not activate and which, thanks to its large spin, would be expected to strongly bind with monopoles which would range out and stop within the array [24]. An initial MMT test array comprising 11 boxes each containing 18 rods of $60 \mathrm{~cm}$ length and $2.5 \mathrm{~cm}$ diameter was installed in September 2012 and exposed to $0.75 \mathrm{fb}^{-1}$ of $8 \mathrm{TeV}$ proton-proton collisions. The boxes were stacked behind the LHCb VELO vacuum vessel just under the beam pipe $1.8 \mathrm{~m}$ away from the interaction point, covering $1.3 \%$ of the total solid angle (see left picture in Fig. 1). After the run is finished, the rods are retrieved and cut to be analysed with a superconducting magnetometer. Only the rods of Box 11 could be cut and scanned in time for this conference. The sample length for this box was a mix of 10, 15, 20 and $30 \mathrm{~cm}$. This measurement serves as a first test of the procedure and as a determination of the optimum sample length for the remaining boxes.

A DC-SQUID rock magnetometer housed at the Laboratory for Natural Magnetism (ETH Zurich) was used. Previous studies performed with rocks and with a small set of material samples from the LHC accelerator demonstrated that this instrument has the capability to detect monopoles trapped in matter with charges much less and much larger than the Dirac charge [22, 25]. The calibration was performed with a convolution method applied to a dipole sample, and cross-checked using thin solenoids which mimic a monopole of well-known magnetic charge [22]. The magnetometer response was found to be linear and charge symmetric. This allows to express the measured currents in units of Dirac magnetic charge.

Each of the 66 samples comprising Box 11 was passed at least once through the magnetometer during a 1-day endeavour in May 2013 (see right picture in Fig. 1). Every tenth measurement on 

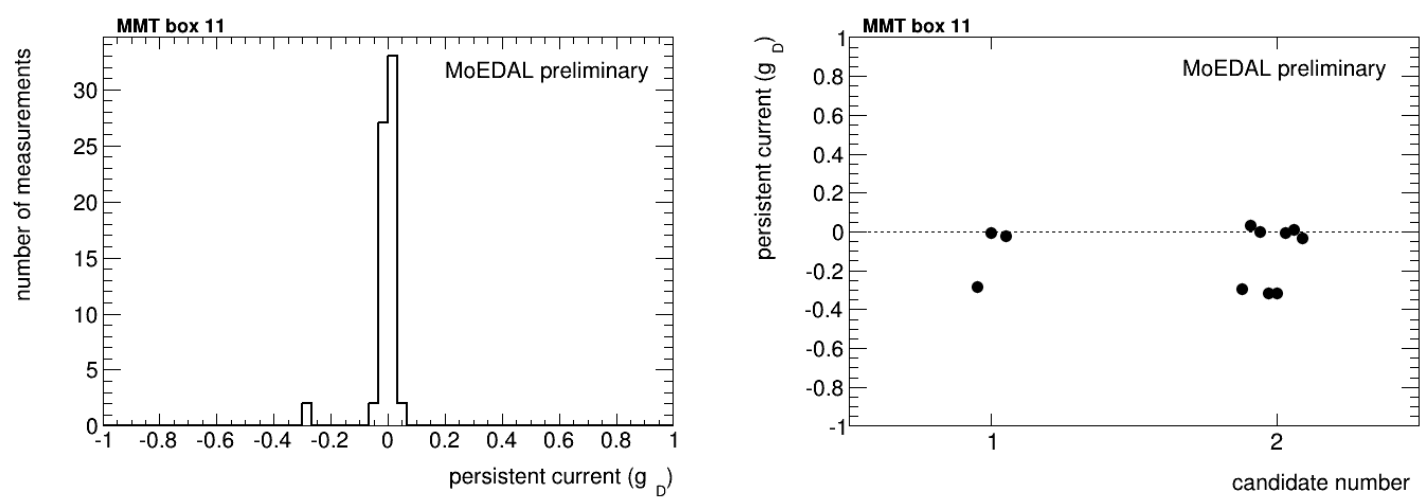

Figure 2: Left: Persistent current (in units of the Dirac charge) obtained with the first measurement of the 66 aluminium samples of Box number 11 of the 2012 MMT test array. Right: Results of multiple persistent current measurements for the 2 samples which yielded large $\left(>0.25 g_{D}\right)$ values for the first measurement.

average is performed with an empty sample holder for the offset subtraction. The relevant measured value is the persistent current, defined as the difference between the currents measured after and before passage of the sample through the sensing coil, and then subtracting the difference obtained with a nearby empty holder measurement. Whenever the persistent current differs from zero by more than one fourth of a Dirac charge, the sample is considered a candidate and measured again several times. A sample containing a genuine monopole would consistently yield the same value for repeated measurements, while values consistent with zero are expected whenever an instrumental effect occurred in the first measurement.

\section{Results of magnetometer measurements}

The magnetic charge contained in the 66 samples of the MMT test array as measured by the first measurement is shown in Fig. 2 (left). No difference was noticed in the practicality and quality of measurements for samples of length 10,15 and $20 \mathrm{~cm}$. The $30 \mathrm{~cm}$ samples were however more difficult to handle due to their large weight. The resolution is in general good for all sample lengths except for two outliers around $-0.3 g_{D}$. Both are are among the large samples $(30 \mathrm{~cm}$ length). Multiple measurements of these potential candidates, shown in the right plot in the figure, are consistent with a zero magnetic charge. The occasional offset jumps to a value around $-0.3 g_{D}$ could be due to the magnetometer entering an abnormal mode, as discussed in Ref. [22]. This effect did not appear when the candidate samples shown here were remeasured during a subsequent campaign in September 2013 where more care was given in resetting the current offset to a value near zero between measurements [26].

\section{Interpretation of the results}

The amount of material between the interaction point and the MoEDAL arrays is typically less than that in front of EM calorimeters in general-purpose detectors, providing sensitivity to higher 
magnetic charges and lower energies [23, 20]. Using an average ${ }^{1}$ of $14 \mathrm{~g} \cdot \mathrm{cm}^{-2}$ of steel for the material of the VELO chamber in front of the MMT test array and $23 \mathrm{~g} \cdot \mathrm{cm}^{-2}$ of aluminium for the array itself, calculations of monopole stopping in the material [20] show that the full MMT test array would trap more than $0.5 \%$ of monopoles with charge between 1 and 4 Dirac charges and mass up to $3500 \mathrm{GeV}$ when assuming a kinematic distribution consistent with a Drell-Yan pair production model. By comparison, the ATLAS published results for $7 \mathrm{TeV}$ collisions [21] — until now the only existing constraints on monopoles at the LHC - are quoted only for one single Dirac charge and a mass up to $1500 \mathrm{GeV}$.

\section{Conclusions and outlook}

MoEDAL is designed for passive detection of magnetic monopoles, both in-flight (with the track-etch technique) and trapped (with the induction technique). A magnetic monopole trapping (MMT) test array was exposed to $0.75 \mathrm{fb}^{-1}$ of $8 \mathrm{TeV}$ proton-proton collisions in 2012. One of the 11 boxes comprising this array was cut into samples of various sizes and scanned with a superconducting magnetometer. No monopoles were found in any of the samples. A sample length of 20 $\mathrm{cm}$ was found to be optimum to minimise the number of samples while keeping good measurement conditions and quality. The remaining boxes were therefore cut into $20 \mathrm{~cm}$ samples and their scanning continued during Summer 2013.

Despite a small solid angle coverage and modest luminosity, the MoEDAL MMT subdetector allows to probe ranges of charge, mass and energy which are not currently accessible to other experiments. Furthermore, this technique can yield results very quickly and would allow to perform a robust, background-free assessment of a signal, potentially providing a direct measurement of a monopole magnetic charge based on its electromagnetic properties only. The full-scale MMT array will be more than 4 times larger than the test array and will be deployed in $2015-2016$ along the back and rear of the LHCb VELO vessel as well as under the floor below the interaction point. This experiment is expected produce the first monopole search results in $14 \mathrm{TeV}$ collisions.

\section{Acknowledgements}

This work was supported by a fellowship from the Swiss National Science Foundation and a grant from the Marc Birkigt Funds of the Geneva Academic Society.

\section{References}

[1] P. Dirac, Quantised Singularities in the Electromagnetic Field, Proc. Roy. Soc. A 133 (1931) 60.

[2] G. 't Hooft, Magnetic Monopoles in Unified Gauge Theories, Nucl. Phys. B 79 (1974) 276.

[3] A. Polyakov, Particle Spectrum in the Quantum Field Theory, JETP Lett. 20 (1974) 194.

[4] Y. Cho and D. Maison, Monopole configuration in Weinberg-Salam model, Phys. Lett. B 391 (1997) 360, arXiv: 9601028 [hep-th].

\footnotetext{
${ }^{1}$ More work needs to be done to precisely quantify the material distribution in the cavern and estimate uncertainties due to the approximations made, which will allow to quote cross-section limits.
} 
[5] T. Kirkman and C. Zachos, Asymptotic Analysis of the Monopole Structure, Phys. Rev. D 24 (1981) 999.

[6] J. Schwinger, Magnetic charge and the charge quantization condition, Phys. Rev. D 12 (1975) 3105.

[7] S. Ahlen, Stopping-power formula for magnetic monopoles, Phys. Rev. D 17 (1978) 229.

[8] S. Ahlen, Theoretical and experimental aspects of the energy loss of relativistic heavily ionizing particles, Rev. Mod. Phys. 52 (1980) 121.

[9] S. Ahlen and K. Kinoshita, Calculation of the stopping power of very-low-velocity magnetic monopoles, Phys. Rev. D 26 (1982) 2347.

[10] M. Fairbairn, A. Kraan, D. Milstead, T. Sjostrand, P. Skands, and T. Sloan, Stable massive particles at colliders, Phys. Rept. 438 (2007) 1, arXiv: 0611040 [hep-ph ].

[11] OPAL Collaboration, Search for Dirac magnetic monopoles in $e^{+} e^{-}$collisions with the OPAL detector at LEP2, Phys. Lett. B 663 (2008) 37, arXiv:0707.0404 [hep-ex] .

[12] CDF Collaboration, Direct search for Dirac magnetic monopoles in p $\bar{p}$ collisions at $\sqrt{s}=1.96 \mathrm{TeV}$, Phys. Rev. Lett. 96 (2006) 201801, arXiv:0509015 [hep-ex].

[13] J. Pinfold, Searching for the magnetic monopole and other highly ionizing particles at accelerators using nuclear track detectors, Radiat. Meas. 44 (2009) 834.

[14] K. Kinoshita, R. Du, G. Giacomelli, L. Patrizii, F. Predieri, P. Serra, M. Spurio, and J. Pinfold, Search for highly ionizing particles in $e^{+} e^{-}$annihilations at $\operatorname{sqrt}(s)=91.1 \mathrm{GeV}$, Phys. Rev. D 46 (1992) 881.

[15] J. Pinfold, R. Du, K. Kinoshita, B. Lorazo, M. Regimbald, and B. Price, A Search for highly ionizing particles produced at the OPAL intersection point at LEP, Phys. Lett. B 316 (1993) 407.

[16] M. Bertani, G. Giacomelli, M. Mondardini, B. Pal, L. Patrizii, F. Predieri, P. Serra-Lugaresi, G. Sini, M. Spurio, V. Togo, and S. Zucchelli, Search for Magnetic Monopoles at the Tevatron Collider, Europhys. Lett. 12 (1990) 613.

[17] H1 Collaboration, A direct search for stable magnetic monopoles produced in positron-proton collisions at HERA, Eur. Phys. J. C 41 (2005) 133, arXiv: 0501039 [hep-ex] .

[18] G. Kalbfleisch, K. Milton, M. Strauss, L. Gamberg, E. Smith, and W. Luo, Improved Experimental Limits on the Production of Magnetic Monopoles, Phys. Rev. Lett. 85 (2000) 5292, arXiv:0005005 [hep-ex].

[19] G. Kalbfleisch, W. Luo, K. Milton, E. Smith, and M. Strauss, Limits on production of magnetic monopoles utilizing samples from the DO and CDF detectors at the Tevatron, Phys. Rev. D 69 (2004) 052002, arXiv:0306045 [hep-ex].

[20] A. De Roeck, A. Katre, P. Mermod, D. Milstead, and T. Sloan, Sensitivity of LHC experiments to exotic highly ionising particles, Eur. Phys. J. C 72 (2012) 1985, arXiv:1112.2999 [hep-ph] .

[21] ATLAS Collaboration, Search for magnetic monopoles in sqrt(s) $=7 \mathrm{TeV}$ pp collisions with the ATLAS detector, Phys. Rev. Lett. 109 (2012) 261803, arXiv:1207.6411 [hep-ex] .

[22] A. De Roeck, H.-P. Hächler, A. M. Hirt, M. Dam Joergensen, A. Katre, P. Mermod, D. Milstead, and T. Sloan, Development of a magnetometer-based search strategy for stopped monopoles at the Large Hadron Collider, Eur. Phys. J. C 72 (2012) 2212, arXiv:1206.6793 [physics.ins-det] .

[23] MoEDAL Collaboration, Technical Design Report of the Moedal Experiment, CERN-LHCC-2009-006 ; MOEDAL-TDR-001 (2009) . 
[24] K. Milton, Theoretical and experimental status of magnetic monopoles, Rep. Prog. Phys. 69 (2006) 1637, arXiv:0602040 [hep-ex].

[25] K. Bendtz, D. Milstead, H.-P. Hachler, A. Hirt, P. Mermod, P. Michael, T. Sloan, C. Tegner, and S. Thorarinsson, Search for magnetic monopoles in polar volcanic rocks, Phys. Rev. Lett. 110 (2013) 121803, arXiv:1301.6530 [hep-ex].

[26] P. Mermod, K. Bendtz, A. Katre, D. Lacarrere, D. Milstead, J. Pinfold, and R. Soluk, Search in 8 TeV pp collisions with the MoEDAL monopole-trapping test array, to appear in proceedings of 14th ICATPP Conference (2013) . 